\title{
A Comparison of Organizational Climate and Nurses' Intention to Leave Among Excellence Awarded Hospitals and Other Hospitals in 2013
}

\author{
Maryam Mohamadzadeh Nojehdehi ${ }^{1}$; Mansoureh Ashgholi Farahani ${ }^{2,}$; Forough Rafii ${ }^{2}$; \\ Nasser Bahrani ${ }^{3}$ \\ ${ }^{1}$ School of Nursing and Midwifery, Iran University of Medical Sciences, Tehran, IR Iran \\ ${ }^{2}$ Center for Nursing Care Research, Iran University of Medical Sciences, Tehran, IR Iran \\ ${ }^{3}$ Statistical Consultant at University of Tehran and Iran Civil Aviation Technology College, Tehran, IR Iran \\ ${ }^{*}$ Corresponding Author: Mansoureh Ashgholi Farahani, Center for Nursing Care Research, Iran University of Medical Sciences, Tehran, IR Iran. Tel:+98-2143651616, E-mail: Farahani. \\ ma@iums.ac.ir; M_negar110@yahoo.com
}

Received: April 13, 2014; Revised: November 18, 2014; Accepted: April 18, 2015

\begin{abstract}
Background: Human resource is the most important factor of performance, success and better revelation of excellence goals of each organization. By performing excellence plan, healthcare organizations improve their organizational climate and play a valuable role in retaining nurses and improving the quality of their services to patients.

Objectives: The aim of this study was to compare hospital organizational climate and intention to leave among working nurses in hospitals performing the excellence plan and other hospitals of Tehran University of Medical Sciences.

Patients and Methods: This was a cross-sectional descriptive comparison study. Its population included 248 nurses of the hospitals performing the excellence plan and other hospitals of Tehran University of Medical Sciences in Iran selected by random sampling. The used instrument had three parts: the first part was related to personal characteristics, the second part was the Munn's organizational climate questionnaire and the third part was Hinshaw's questionnaire of "anticipated turnover scale". Data was analyzed using SPSS software, version 17 and indices of descriptive statistics and inferential statistics.

Results: The results of the mean and standard deviation for organizational climate and intention to leave in both performing and nonperforming hospitals of the organizational excellence plan were respectively (65.28 \pm 19.31 and $56.42 \pm 21.36)$ and (33.64 \pm 5.58 and $35.59 \pm$ 4.94). Independent $\mathrm{T}$ test revealed a significant difference between the mean scores for organizational climate in both performing and non-performing hospitals, and also a significant difference between the mean scores for intention to leave in both performing and nonperforming hospitals $(\mathrm{P}=0.004)$. Moreover, Pearson Correlation test showed a reverse significant correlation between organizational climate and intention to leave in performing hospitals of the organizational excellence plan $(r=-0.337)$ and non-performing hospitals $(r$ $=-0.282)(\mathrm{P}=0.001)$.

Conclusions: Performing quality improvement pattern such as organization's excellence plan improves organizational climate of healthcare sectors, it can reduce nurses' intentions to leave and retain human resources.
\end{abstract}

Keywords: Organizational Culture; Climate; Intention; Attitude; Total Quality Management; Nurses

\section{Background}

Regarding the importance of human resources, employees' intension to leave the organization is one of the concerns of human resources management in organizations (1). Some researchers believe that intention to leave is a behavioral approach, since a person's idea or intention is the strongest determining factor of selection and a person's behavior is subordinate to their intention to do that particular behavior, the intention to leave is highly correlated with leaving the job $(2,3)$. Therefore, organizations are always concerned about losing their human capitals. Each organization spends a lot of money on education and preparation of its employees until reaching to the stage of profitability and desirable efficiency. Swansburgh indicated that $60 \%$ of the total budget of hospitals is spent on hiring and educating their employees; therefore, by leaving their valuable staff, hospitals lose skills and experiences gained through years (4).

On the other hand, the results of other researches showed that nurses' intention to leave would cause many problems in presenting services to patients (5), quality of care (6) and treatment costs (7). The strong intentions to leave among nurses considered as the most important factor in the organization prevent the organizations from reaching their organizational goals (8). Lutzen et al. showed that $71.42 \%$ of nurses think about leaving their professions everyday (9).

The goal of healthcare organizations and managers of studying the issue of intention to leave as a potential fac-

Copyright ( 2015, Iranian Red Crescent Medical Journal. This is an open-access article distributed under the terms of the Creative Commons Attribution-NonCommercial 4.0 International License (http://creativecommons.org/licenses/by-nc/4.0/) which permits copy and redistribute the material just in noncommercial usages, provided the original work is properly cited. 
tor is to prevent real leaving (10). One of the important and effective organizational factors in reducing nurses' intention to leave is the organizational climate (11). In different and sensitive environment of hospitals, desirable organizational climate significantly affects many of employees' behaviors in achieving excellence organizational goals. Improper organizational climate can be a major source of psychological pressure and if these pressures exceed the allowed limit, they can increase employees' intention to leave and threaten organizational goals $(12,13)$. Today, the issue of competition in attracting more human resources and presenting high-quality services is one of the important challenges of healthcare system. In this situation, only organizations which pay attention to their employees can survive (14). Therefore activities of human resources management in relation with employees must go beyond the traditional activities of human resources management, which require innovative, flexible and effectual strategies (15-17).

One of the important and effective factors on improvement of organizational climate is using organization's excellence models. In this regard, using organizational excellence plans such as European Foundation for Quality Management (EFQM) and modern management methods, magnet hospitals have been able to increase the feelings of commitment and belonging among nurses as the organization internal customers as a fundamental orientation towards the organizational mission and strategic goals $(1,18,19)$. EFQM excellence model has three levels. At the first level, nine criteria have been considered including five criteria of empowerment and four criteria of results, which are used to evaluate and perform the fundamental concepts and values in the organization. These nine criteria are leadership, policy and strategy, employees (human resources), participations and resources, customers' results, employees' results, society results and performance key results. At the second level, there are 32 subsets for the expression of the meanings of the first level's criteria and at the third level, a list of guidelines has been presented for more explanation of the subset (20). The excellent hospitals focus on human capitals' results and comprehensively measure significant results related with their employees and achieve them. These results show employees' satisfaction and available trends of this section. This section evaluates both employees' efficient perceptions (which may be resulted from investigations, focus groups, interviews, etc.) and the internal performance indices (such as efficiency, intention to leave, illness level, early retirement and accident levels) (20). To create a positive organizational climate, excellent hospitals develop employees' retention strategies and enable themselves to achieve loyalty, trust and survival through concentration on the potential and actual demands and expectations of the internal customers (21). Therefore, implementing structural modification programs in hospitals alike implementing quality improvement programs or implementing EFQM model, results in the improvement of workplace conditions and organizational climate, significant increase of organization performance and better revelation of the organization excellence goals $(22,23)$.

\section{Objectives}

Therefore, describing and comparing organizational climate and intention to leave between nurses of hospitals performing excellence plan and other hospitals, the present research aimed to determine the association between these two variables in the mentioned centers.

\section{Patients and Methods}

This was a descriptive-comparative study. Its statistical population was all nurses working in the hospitals. Regarding studies conducted inside and outside Iran and the general objective of the research, the samples of the research were calculated 248 individuals at the confidence interval of $95 \%$ and the power of $90 \%$, while considering the mean and standard deviation of the variable "intention to leave" as respectively 2.59 and 0.55 at magnet hospital and 2.36 and 0.7 at ordinary hospitals (these values are reported at studies of Stordeur et al. (1) and Hariri et al. (24). The researchers used the formula (Equation 1) to calculate the number of samples. Then, the stratified random sampling method was used in each hospital to select participants.

1)

$$
n=\frac{\left(Z_{1-\frac{\alpha}{2}}+Z_{1-\beta}\right)^{2}\left(S_{1}^{2}+S_{1}^{2}\right)}{\left(m_{1}-m_{2}\right)^{2}}
$$

Table 1. The Frequency Distribution of Nurses Under Study in Each Affiliated Hospital to Tehran University of Medical Sciences Regarding Performance of Organizational Excellence Plan ( $\mathrm{n}=$ $248)^{\mathrm{a}}$

\begin{tabular}{|lc|}
\hline Variables & Values \\
\hline $\begin{array}{l}\text { Hospitals performing the Organiza- } \\
\text { tional Excellence Plan }\end{array}$ \\
\hline Roozbeh & $23(18.7)$ \\
\hline Jame Zanan & $23(18.7)$ \\
\hline Hashemi Nejad & $31(25.2)$ \\
\hline Farabi & $46(37.4)$ \\
\hline Total & 123 \\
\hline
\end{tabular}

Hospitals not performing the Organi-

zational Excellence Plan

\begin{tabular}{lc} 
Firoozgar & $36(28.8)$ \\
Shahid Akbar Abadi & $16(12.8)$ \\
Hazrat Rasool & $60(48)$ \\
Tehran Psychology & $13(10.4)$ \\
Total & 125 \\
\hline a Data are presented as No. (\%)
\end{tabular}

a Data are presented as No.(\%). 
The distribution of nurses in the study in each of the hospitals regarding performance of organizational excellence plan is displayed in Table 1.

Nurses who directly took care of patients with at least B.Sc. degree and working fulltime in three working shifts of morning, afternoon and night in the mentioned hospitals at least for six months allowed to be recruited. The exclusion criterion was unwillingness for participation. The research environment included eight teaching, public and referral hospitals affiliated to the Tehran University of Medical Sciences, from which four performed organizational excellence plan (Jame Zanan Hospital with 118 beds, Roozbeh Psychiatric Hospital with 165 beds, Farabi Eye hospital with 199 beds and Hashemi Nejad Kidney and urinary tract hospital with 163 beds), and four did not perform the organizational excellence plan (Akbar Abadi Obstetrics and Gynecology Hospital with 267 beds, Iran Psychiatric hospital with 105 beds, Hazrat Rasool general hospital with 426 beds and Firoozgar general hospital with 192 beds) in 2013 (www.research.ac.ir). The hospitals performing the organizational excellence plan in this research implemented the EFQM model, and after evaluating by the Institute for Studies in Human Resource and Productivity, were selected as the best organizations (http://www. iranaward.org).

These centers were identified in demographic questionnaires by the researcher. The data collection tool of this research was a questionnaire related to nurses' demographic characteristics with 11 questions on gender, age, level of education, job background, etc.

The other tool used in the research was the Munn's organizational climate questionnaire including 25 Likert questions, scored 0 to 4 (never, rarely, sometimes, often and always). Based on the standard measures, the scores were calculated from 100, 90 - 100 as excellent organizational climate, 80 - 90 as good organizational climate, 70 - 79 as medium, 60 - 69 as relatively weak and below 60 as weak (4).

This questionnaire was translated into Persian in 2005 by Nik Peyma. The validity and reliability of this questionnaire were assessed respectively by content credit method and test-retest and the reliability score of the test was higher than $80 \%$. The validity of this questionnaire was tested in another study of Nik Peyma in 2010 using testretest validity method and the value was calculated to be 0.84 (25). The Alfa Coefficient in the present study is considered to $b(\alpha=0.92)$.

The other tool used in this research was Hinshaw's questionnaire of anticipated turnover scale including 12 Likert questions ranging from 1 to 5 ( 5 for complete agreement, and 1 for complete disagreement). The total score ranged from 5 to 60 ; the final score is calculated by dividing the sum of scores over the number of questions that is $12(26)$. After the researcher studied the guide, different levels of intention to leave were calculated based on the mean and standard deviation of the total score for intention to leave among the nurses. The intention to leave was considered strong for scores above 39, medium for scores 29 to 39 and weak for below 29. The questionnaire's reliability was confirmed in the study of Hariri et al. (24), and its validity was calculated as $80 \%$ by means of Cronbach's alpha coefficient. The method of questionnaire completion by nurses was self-reporting. Ethics committee of Tehran University of Medical Sciences approved the study (No. 91/D/ 130/3169 in 2/3/2013). The study was performed by considering moral aspects like conscious willingness of participants, observing confidentiality and assuring participants to keep their information and identity secure. After data collection, SPSS version 17 was used to analyze data as well as descriptive statistics tools including tables and central indices like mean, distribution indices like standard deviation, inferential statistical tests like independent T-test for comparing means, and Pearson correlation coefficient for assessing the correlation between organizational climate and intention to leave in different hospitals regarding performance of the organizational excellence plan. $\mathrm{P}<0.05$ was considered significant.

\section{Results}

Demographic characteristics of the research participants are shown in Table 2.

Kolmogorov-Smirnov test showed normal distribution of data, consequently, parametric statistical tests were used.

The results showed that in both groups of hospitals, most nurses aged below 30, most of them were female and married, more than $90 \%$ had B.Sc. degrees, the employment status of most nurses in hospitals that performed the organizational excellence plan was $46.3 \%$ as permanent recruitment, and $56 \%$ as contract employment, the working experience of more than $50 \%$ of nurses was lower than 10 years, $73.3 \%$ of nurses in the hospitals that performed the excellence plan and 55.3\% of nurses in the hospitals that did not perform the plan had changing shifts. The required weekly working hours for most nurses in the study was lower than 50 hours. $84 \%$ of nurses were not willing to work overtime in the hospitals that performed the organizational excellence plan and $62.6 \%$ in the hospitals that did not perform the plan. The results showed that $80.5 \%$ of nurses in the hospitals that performed the organizational plan and $60 \%$ of the nurses working in the hospitals that did not perform the plan liked their organization.

Based on the findings in Table 3, independent T-test showed a significant difference between the means of organizational climate in performing and non-performing hospitals of organizational excellence plan ( $\mathrm{P}=0.001)$. The above table shows that hospitals that performed organizational excellence plan had a better climate than hospitals that did not perform this plan. 
Table 2. The Frequency Distribution of Most Common Demographic Characteristics of Nurses in Each Hospital Regarding Performance of Organization's Excellence Plan Affiliated to Tehran University of Medical Sciences in $2013(\mathrm{n}=248)^{\mathrm{a}}$

\begin{tabular}{lccc}
\hline Demographic Characteristics & Groups & \multicolumn{2}{c}{ Treatment Centers } \\
\cline { 3 - 4 } & & $\begin{array}{c}\text { Not Performing the Orga- } \\
\text { nization Excellence Plan }\end{array}$ & $\begin{array}{c}\text { Performing the Organiza- } \\
\text { tion Excellence Plan }\end{array}$ \\
\hline Age Groups & Less than 30 & $53(42.4)$ & $45(36.6)$ \\
Gender & Female & $121(96.8)$ & $104(84.6)$ \\
\hline Marital Status & Married & $85(68)$ & $91(74)$ \\
\hline Education & B.Sc. & $117(93.6)$ & $118(95.9)$ \\
\hline Employment Status & Stipulated in a Contract & $70(56)$ & $40(32.5)$ \\
\hline Job Background & Less than 10 years & $83(66.4)$ & $67(54.5)$ \\
\hline Shift Type & Shifting & $92(73.6)$ & $68(55.3)$ \\
Compulsory Working Hours & Less than 50 Hours & $61(48.8)$ & $94(76.4)$ \\
\hline Overtime Hours & Between 50 - 100 Hours & $44(35.2)$ & $77(62.6)$ \\
\hline Tendency to Work Overtime & No & $105(84)$ & $99(80.5)$ \\
\hline Liking the Organization & Yes & $75(60)$ & \\
\hline
\end{tabular}

a Data are presented as No. (\%).

Table 3. Comparing the Organizational Climate in the Studied Affiliated Hospitals to Tehran University of Medical Science That Performed and Did not Perform the Organizational Excellence plan in $2013(n=248)^{a}$

\begin{tabular}{lcc}
\hline Hospitals & \multicolumn{1}{c}{ The Organization's Excellence Plan } \\
\hline Organizational Climate & Not-Performed & Performed \\
\hline Weak (lower than 60) & $72(57.6)$ & $41(33.3)$ \\
\hline Relatively Weak (between 60 - 69) & $15(12)$ & $22(17.9)$ \\
\hline Medium (between 70-79) & $17(13.6)$ & $33(26.8)$ \\
\hline Good (between 80-89) & $9(7.2)$ & $12(9.8)$ \\
Excellent (between 90-100) & $9(7.2)$ & $12(9.8)$ \\
Total & $125(100)$ & $65.28 \pm 19.31$ \\
\hline X \pm SD & $56.42 \pm 21.36$ & \\
\hline Independent T-Test & $\mathrm{T}=3.418 ; \mathrm{P}=0.001 ; \mathrm{df}=246$ &
\end{tabular}

Table 4. The Frequency Distribution of Intention to Leave Among Nurses of Hospitals Performing/Not Performing the Organization Excellence Plan in $2013(\mathrm{n}=248)^{\mathrm{a}}$

\begin{tabular}{lcc}
\hline \multirow{2}{*}{ Intention to Leave } & \multicolumn{2}{c}{ Hospital } \\
\cline { 2 - 3 } Weak & Not Performing the Excellence Plan & Performing the Excellence Plan \\
\hline Medium & $14(11.2)$ & $31(25.2)$ \\
Strong & $84(67.2)$ & $69(56.1)$ \\
Total & $27(21.6)$ & $23(18.7)$ \\
$\mathbf{X} \pm$ SD & $125(100)$ & $33.64 \pm 5.58$ \\
Independent T-Test & $35.59 \pm 4.94$ & \\
\hline a Data are presented as No. or No. (\%) or mean $\pm S D$. & $\mathrm{T}=2.892 ; \mathrm{P}=0.004 ; \mathrm{df}=246$
\end{tabular}


Table 5. Correlation Between Organizational Climate and Intention to Leave in Hospitals Performing/Not Performing the Organization Excellence Plan in 2013

\begin{tabular}{lcccc}
\hline Variable & Hospitals Performing the Excellence Plan & Hospitals not Performing the Excellence Plan \\
\cline { 2 - 5 } & $\mathbf{P}$ & $\mathbf{r}$ & $\mathbf{P}$ & $\mathbf{r}$ \\
\hline Intention to Leave & & & & -0.282 \\
\hline Organizational Climate & 0.001 & -0.337 & 0.001 & \\
\hline
\end{tabular}

Based on the findings in Table 4, independent T-test showed a significant difference between the score means of intention to leave the organization in performing and non-performing organizations of the excellence plan ( $\mathrm{P}$ $=0.004)$. The findings showed that performing the organizational excellence plan reduced the intention to leave the organization in performing hospitals of this plan.

According to Table 5, Pearson Correlation test showed a significant and inverse association between organizational climate and the score of intention to leave $(\mathrm{P}=0.001)$.

\section{Discussion}

The findings of this research are in accordance with other studies' results stated below. Liou and Cheng reported a significant negative association between organizational climate and intention to leave $(P<0.001)(27)$. Moreover, the present study showed that organizational climate (60\%) indirectly affects organizational commitments and intention to leave and creation of a positive organizational climate can increase nurses' commitment to their job and reducing their intention to leave.

Furthermore, the results of the study performed by Kutney-Lee et al. (28) in Pennsylvania showed a significant and inverse association between improvement of nurses' workplace and their intention to leave $(\mathrm{P}<0.01)$. The results of this study confirmed and supported the implementation of EFQM plan in hospitals; they along with the nursing accreditation center of the United States, provide healthcare organizations with a plan which results in improvement of leadership and empowerment, professional performance and improvement of workplace for nurses. The performed studies showed that creation of an excellent organizational climate as a fundamental orientation towards the organization's organizational missions and strategic goals increases employees' satisfaction and creates better conditions for nurses' interactions and reduces intention to leave among them.

Establishment of organization excellence plan to improve management and quality level attracted the attention of healthcare system. The results showed that achieving this goal requires more improvement of organizational climate and extensive participation of all employees towards constant improvement and correctional and innovative thinking. Moving organizations towards accomplishing organizational excellence goals would be very difficult, if it is not in line with perceptions and beliefs of that organization employees (29).
Practically speaking, hospitals performing the excellence plan employ new management strategies such as employees' participation in decisions and basing the evaluation system on performances (21). The results of the study performed by Van der Heijden et al. (10) in hospitals of Belgium and Stordeur et al. (1) indicate this subject as well. It can be concluded that in attractive hospitals, the organizational climate is such that the employees really trust each other. Furthermore, due to the balance between performance and rewards, the amount of nurses' job satisfaction is higher compared with other hospitals. In such organizations, nurses actively participate to improve hospital excellence goals. In hospitals with plain organizational structures, the participatory management method, structured educational programs and opportunities of job improvement can retain nurses in the healthcare system. On the contrary, in hospitals with weak management, nurses can be retained less (3, 21). Improvement of organizational characteristics based on using organization excellence models such as EFQM and accreditation programs in hospitals are valuable strategies in reducing intention to leave. In the competitive environments among organizations, each organization tries to improve the quality of its services. On the other hand, orienting the organizations towards accomplishing organizational excellence goals would be a difficult task without committed human resource.

Organizational climate is a modifiable organizational variable in hospitals and is of important factors leading to unpleasant effects such as intention to leave their workplace and eventually leave this profession. Therefore, to improve the quality of performance in hospitals and achieve organization excellence goals (presenting safe and standard healthcare services to patients, recognizing hospital's necessary leadership style and implementing them, improving job satisfaction, creating organizational commitment, reducing employees' intention to leave, improving treatment quality, etc.), the managers and those who are responsible for nursing must improve the organizational climate of healthcare centers by focusing more on human capitals as one of the empowering criteria of organization excellence plan through different methods such as fairly distributing rewards based on job quality and employees' attempts in performing hard tasks, allowing employees to participate in mission formulation and organizational goals and therefore, providing necessary conditions and opportunities for their growth, making them participate in decision making, 
welcoming new and creative ideas, showing sensitivity to their personal likes and opinions, etc., and providing the situation to achieve employees' best results in organization excellence plan.

\subsection{Research Limitations}

This research was conducted on employed nurses in affiliated hospitals to Tehran University of Medical Sciences, consequently, generalizing the results of this research to other organizations must be with cautious.

\section{Acknowledgements}

Hereby, the researchers thank the managers of the selected hospitals and those nurses who participated in the performance of this research. This article is the research plan approved by Tehran University of Medical Sciences. The budget of this research was provided by the research assistance of Tehran University of Medical Sciences.

\section{Authors' Contributions}

Study concept and design: Maryam Mohamadzadeh Nojehdehi, Mansoureh Ashgholi Farahani and Forough Rafii; Data Gathering: Maryam Mohamadzadeh Nojehdehi; statistical analysis and interpretation of data: Naser Bahrani and Maryam Mohamadzadeh Nojehdehi; drafting of the manuscript: Maryam Mohamadzadeh Nojehdehi and Mansoureh Ashgholi Farahani.

\section{Funding/Support}

This study was supported by Iran University of Medical Sciences (IUMS/SHMIS-Dec/2011/132).

\section{References}

1. Stordeur S, D'Hoore W, N. EXT-Study Group . Organizational configuration of hospitals succeeding in attracting and retaining nurses. J Adv Nurs. 2007;57(1):45-58.

2. Volk MC, Lucas MD. Relationship of management style and anticipated turnover. Dimens Crit Care Nurs. 1991;10(1):35-40.

3. Tanaomi M. Intention to leave and Turnover action. Knowl Manag. 1993:5-21.

4. Swansburg RJ. Introduction to management and leadership for nurse managers.: Jones \& Bartlett Learning; 2002.

5. Clarke SP, Aiken LH. Failure to Rescue: Needless deaths are prime examples of the need for more nurses at the bedside. Am J Nurs. 2003;103(1):42-7.

6. Aiken LH, Clarke SP, Sloane DM, Sochalski J, Silber JH. Hospital nurse staffing and patient mortality, nurse burnout, and job dissatisfaction. JAMA. 2002;288(16):1987-93.

7. Rivers PA, Tsai KL, Munchus G. The financial impacts of the nursing shortage. J Health Care Finance. 2004;31(3):52-64.

8. Newman K, Maylor U, Chansarkar B. "The nurse satisfaction, service quality and nurse retention chain": implications for management of recruitment and retention. J Manag Med.2002;16(4-5):271-91.

9. Lutzen K, Blom T, Ewalds-Kvist B, Winch S. Moral stress, moral climate and moral sensitivity among psychiatric professionals. Nurs Ethics. 2010;17(2):213-24.

10. van der Heijden BI, Kummerling A, van Dam K, van der Schoot E, Estryn-Behar M, Hasselhorn HM. The impact of social support upon intention to leave among female nurses in Europe: secondary analysis of data from the NEXT survey. Int J Nurs Stud. 2010;47(4):434-45.

11. Saatchi.. Efficiency psychology.Tehran: virayesh; 2000.

12. Kermani.. [Relationship between organizational climate and employees' organizational commitment in Hamadan University of Medical Sciences].Tehran: Islamic Azad University, Research and Sciences Branch; 2008.

13. Kadiwar P. Relationship between high school students' ethical Jugements development with organizational climate. Educ J. $1994 ; 48$.

14. Mardan M, Hamule M, Shahraki Vahed A. [Relationship between hospital personnel's personality characteristics and organizational climate, Najafabad, Iran]. J Health Admi. 2010;12(38):23-30.

15. Amirianzadeh M. [A study of organizational climate and job satisfaction relationship in Shiraz education office].Shiraz: Eslamic Azad University Shiraz Branch; 2004.

16. Torabi A. The relationship between administrators leadership style and conflict management in Lorestan University of Medical Sciences Hospitals.; 2004.

17. Horwitz FM, Heng CT, Quazi HA, Nonkwelo C, Roditi D, Eck PV Human resource strategies for managing knowledge workers: an Afro-Asian comparative analysis. Int J Human Resour Manag. 2006;17(5):775-811.

18. San Park J, Hyun Kim T. Do types of organizational culture matter in nurse job satisfaction and turnover intention? Leadersh Health Serv . 2009;22(1):20-38.

19. Mohabatkhah $\mathrm{H}$. The concept of organizational climate survey. 2011.

20. Homayounfar M. Organizational Excellence Model.3th ed: Excelled publisher First Printing; 2011.

21. Fathi M. . Model of excellence healthcare. Administrative change and Management Office.: Ministry of Health and Medical Education; 2012 .

22. Taslimi M. Organizational change management. 8th edTehran: Samt Publications; 2008.

23. Albaugh JA. Spirituality and life-threatening illness: a phenomenologic study. Oncol Nurs Forum. 2003;30(4):593-8.

24. Hariri G, Shakeri N, Yaghmaei.. [Assessment of some factors related to leave in nurses and their demographic charater in educational hospitals of Shahid Behesthi University of Medical Sciences].J Health Promot Manag. 2012.

25. Nicpeyma N. [Evaluation the relationship between organizational climate and absenteeism among nurses and identify absenteeism causes].Tehran: University of Social Welfare and Rehabilitation Sciences; 2005.

26. Flanagan NA. Testing the relationship between job stress and satisfaction in correctional nurses. Nurs Res. 2006;55(5):316-27.

27. Liou SR, Cheng CY. Organisational climate, organisational commitment and intention to leave amongst hospital nurses in Taiwan. J Clin Nurs. 2010;19(11-12):1635-44.

28. Kutney-Lee A, Wu ES, Sloane DM, Aiken LH. Changes in hospital nurse work environments and nurse job outcomes: an analysis of panel data. Int J Nurs Stud. 2013;50(2):195-201.

29. Translation by Gerami M. NH. [European foundation for quality management. Organizational excellence. Saramad publisher, Dependent on Institute of Human Resource Productivity]Translated by Gerami and Noor Alizadeh, .; 2008. 